\title{
Comparison of olmesartan combined with a calcium channel blocker or a diuretic in elderly hypertensive patients (COLM Study): safety and tolerability
}

\author{
Takao Saruta ${ }^{1}$, Toshio Ogihara ${ }^{2}$, Ikuo Saito ${ }^{1}$, Hiromi Rakugi ${ }^{3}$, Kazuaki Shimamoto ${ }^{4}$, Hiroaki Matsuoka ${ }^{5}$, \\ Satoshi Teramukai ${ }^{6}$, Jitsuo Higaki ${ }^{7}$, Sadayoshi Ito ${ }^{8}$ and Kazuyuki Shimada ${ }^{9}$ for the COLM Investigators
}

The cardiovascular effects of combined therapy with the angiotensin receptor blocker (olmesartan) and a dihydropyridine calcium channel blocker (CCB) or a diuretic were compared in high-risk elderly Japanese hypertensive patients by performing a randomized, open label, blinded-endpoint study of morbidity and mortality (the COLM study). Here we report the results obtained with respect to safety and tolerability. High-risk hypertensive patients aged 65-84 years were enrolled and were randomized to receive olmesartan combined with either a CСB (amlodipine or azelnidipine) or a low-dose diuretic for at least 3 years. The primary endpoint was a composite of fatal and non fatal cardiovascular events, whereas adverse events (AEs) and the percentage of patients who discontinued the allocated treatment were evaluated as secondary endpoints. A total of 5141 patients were randomized. Both combination regimens achieved a similar reduction of cardiovascular morbidity and mortality. The incidences of AEs, serious AEs, drug-related serious AEs and discontinuation due to serious AEs were lower in the olmesartan plus CCB group than in the olmesartan plus diuretic group. Serum levels of uric acid and creatinine were significantly higher in the olmesartan plus diuretic group than in the olmesartan plus CCB group. Olmesartan combined with a CCB was significantly superior to olmesartan plus a diuretic with regard to the frequency of AEs and discontinuation of treatment.

Hypertension Research (2015) 38, 132-136; doi:10.1038/hr.2014.141; published online 25 September 2014

Keywords: adverse event; combination therapy; discontinuation; elderly hypertensive patient; olmesartan

\section{INTRODUCTION}

Hypertension is a major public health problem that is associated with significant cardiovascular morbidity and mortality. There is a growing body of evidence which shows that antihypertensive therapy substantially reduces the incidence of cardiovascular disease, provided that the blood pressure (BP) is controlled tightly. ${ }^{1,2}$ To achieve sufficiently tight BP control, it is often necessary to employ combination therapy with multiple antihypertensive agents of different classes, ${ }^{3-8}$ but the optimum combination has not yet been elucidated. In recent clinical practice, an angiotensin receptor blocker (ARB) combined with a calcium channel blocker (CCB) or an ARB combined with a diuretic have been widely used for the treatment of hypertension. ${ }^{6}$ However, it is still unclear which combination is more beneficial for the prevention of cardiovascular disease, as well as which is better with regard to safety and tolerability. Combination of olmesartan and a CCB or a diuretic in Japanese elderly hypertensive patients (COLM) trial was a prospective, randomized, open-label, blinded-endpoint (PROBE) study to determine which combination is a preferable therapy for hypertension, $\mathrm{ARB}$ plus $\mathrm{CCB}$ or $\mathrm{ARB}$ plus diuretic, ${ }^{9,10}$ and the principal results have demonstrated that there were no remarkable differences in the primary composite endpoints of cardiovascular morbidity and mortality between the two groups, olmesartan plus CCB or diuretic. ${ }^{8}$ However, safety and tolerability profiles suggested that olmesartan plus CCB may be preferable to olmesartan plus diuretic. ${ }^{8}$ In this article, the details of the COLM-study findings with respect to safety and tolerability are reported.

\section{METHODS}

The rationale, design, management and principal results of the COLM study have already been reported. ${ }^{9,10}$

${ }^{1}$ Department of Internal Medicine, School of Medicine, Keio University, Tokyo, Japan; ${ }^{2}$ Morinomiya University of Medical Sciences, Osaka, Japan; ${ }^{3}$ Department of Geriatric Medicine and Nephrology, Osaka University Graduate School of Medicine, Osaka, Japan; ${ }^{4}$ School of Medicine, Sapporo Medical University, Sapporo, Japan; ${ }^{5}$ Department of Hypertension and Cardiorenal Medicine, Dokkyo Medical University, Tochigi, Japan; ${ }^{6}$ Department of Clinical Trial Design and Management, Translational Research Center, Kyoto Prefectural University of Medicine Graduate School of Medical Science, Kyoto, Japan; ${ }^{7}$ Division of Cardiology, Department of Integrated Medicine and Informatics, Ehime University Graduate School of Medicine, Shitsukawa, Ehime, Japan; ${ }^{8}$ Department of Nephrology, Endocrinology, and Vascular Medicine, Tohoku University Graduate School of Medicine, Sendai, Japan and ${ }^{9}$ Department of Cardiology, Shin-Oyama City Hospital, Oyama, Japan

Correspondence: Professor T Saruta, 1003 Koie Building, 6-7 Samon-cho, Sinjuku-ku, Tokyo 160-0017, Japan.

E-mail: takao_saruta@ybb.ne.jp

Some data in this report were presented at the 23rd European meeting on Hypertension and Cardiovascular Protection (Milan, Italy), 15 June 2013.

Received 2 July 2014; revised 14 August 2014; accepted 19 August 2014; published online 25 September 2014 
In brief, hypertensive patients aged 65-84 years, with a history of cardiovascular disease and/or cardiovascular risk factors, who had a systolic $\mathrm{BP} \geqslant 140 \mathrm{~mm} \mathrm{Hg}$ and/or diastolic $\mathrm{BP} \geqslant 90 \mathrm{~mm} \mathrm{Hg}$ while on antihypertensive treatment or a systolic $\mathrm{BP} \geqslant 160 \mathrm{~mm} \mathrm{Hg}$ and/or diastolic $\mathrm{BP} \geqslant 100 \mathrm{~mm} \mathrm{Hg}$ without treatment, were randomized to receive olmesartan plus either a CCB (amlodipine or azelnidipine) or a low-dose diuretic (trichlormethiazide, indapamide or some other thiazide) for at least 3 years. The target BP was $<140 / 90 \mathrm{~mm} \mathrm{Hg}$.

The primary endpoint was the occurrence of fatal or non-fatal cardiovascular events, including sudden death, fatal and non-fatal stroke including transient ischemic attack, fatal and non-fatal cardiac events and renal events.

Secondary endpoints were as follows: cardiovascular death, non-fatal myocardial infarction, non-fatal stroke (all of which cause death) a composite hard endpoint (cardiovascular death, non-fatal myocardial infarction and nonfatal stroke, excluding transient ischemic attack), new-onset of diabetes, the incidence of specific events (sudden death, cerebrovascular events, cardiac events and renal events), new-onset of atrial fibrillation, adverse events (AEs) and the discontinuation rate for each allocated treatment. AEs were classified as drug related or nondrug related and as serious or non serious, and were monitored throughout the study.

All cardiovascular events and serious AEs (SAEs) reported by the participating investigators were adjudicated by the Endpoint committee that was blinded to the study group.

\section{Statistical analysis}

Patient characteristics were reported as mean \pm s.d. or percentage. The frequency rates of AEs were compared by using Fischer's exact test. Student's $t$-test was used to compare the two groups. Time-to-continuation curves were drawn with the Kaplan-Meier method for the continuation rates in each treatment group and the stratified log-rank test was used to compare these rates between the two groups. Repeated measures analysis of variance was used to compare the changes of estimated glomerular filtration rate between the two groups. All statistical analyses were done with SAS 9.1 software (SAS Institute, Cary, NC, USA).

\section{RESULTS}

Study groups and baseline characteristics

Details of the study groups and baseline characteristics were described in the previous report. ${ }^{9}$ In brief, a total of 5658 patients were assessed for eligibility. After the 449 patients who met the exclusion criteria and the 28 patients who did not give consent were

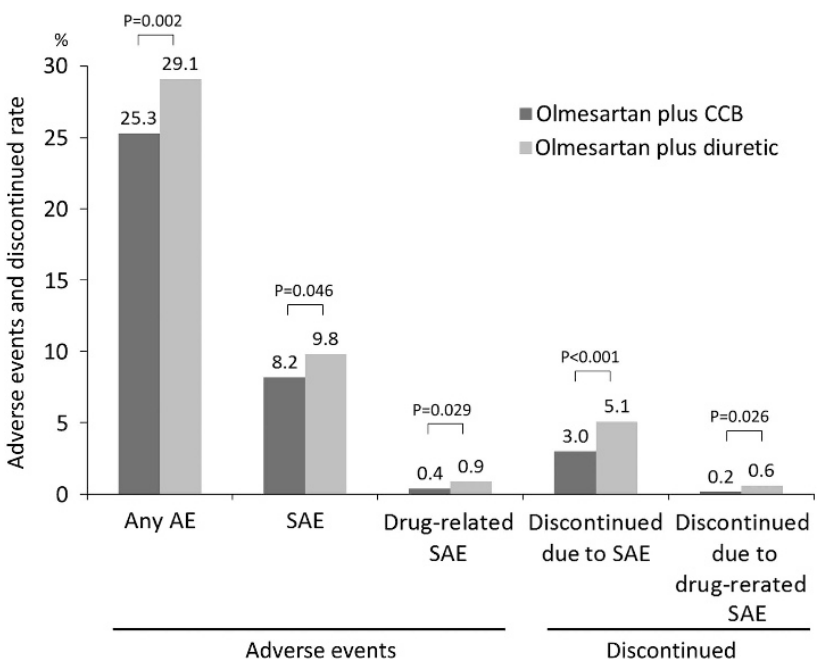

Figure 1 Adverse events and discontinuation rate. Data on AEs and SAEs were reported previously with the principal results. ${ }^{9}$ AEs, adverse events; $\mathrm{CCB}$, calcium channel blocker; SAE, serious adverse events. excluded, the remaining 5141 patients were randomly assigned for treatment with olmesartan plus a CCB (olmesartan plus CCB group) or olmesartan plus a diuretic (olmesartan plus diuretic group). A total of 46 patients in the olmesartan plus CCB group and 72 patients in the olmesartan plus diuretic group were lost to follow-up and 5023 patients (98\%) completed the follow-up period. The mean follow-up period was 3.3 years, the mean age of the patients was 73.6 years and $51.6 \%$ of the subjects were men. There were no significant differences of baseline characteristics between the two treatment groups. About $24 \%$ of the patients had a history of cardiovascular disease, including stroke $(14.6 \%)$ and ischemic heart disease $(11.0 \%)$.

\section{Safety and AEs}

The olmesartan plus CCB group showed a lower incidence of all AEs, SAEs, drug-related SAEs and discontinuation due to SAEs than the olmesartan plus diuretic group (Figure 1). Conversely, the continuation rate was significantly lower in the olmesartan plus diuretic group than in the olmesartan plus $\mathrm{CCB}$ group $(P<0.001$; Figure 2$)$. In addition, the total discontinuation rate was lower in the olmesartan plus CCB group than in the olmesartan plus diuretic group $(20.7 \%$ vs. $32.4 \%, P<0.001)$.

Table 1 summarizes SAEs reported in more than 10 patients from each group. The incidence of fracture (the fourth most frequent SAE) was significantly higher in the olmesartan plus CCB group than in the olmesartan plus diuretic group.

Regarding laboratory data, changes in serum levels of uric acid and creatinine were significantly greater in the olmesartan plus diuretic group than in the olmesartan plus CCB group (for both groups $P<0.001)$. There were significantly more patients with hyperuricemia in the olmesartan plus diuretic group than in the olmesartan plus CCB group (153/2573, 6.5\% vs. 61/2568, 2.6\%; $P<0.001)$. None of the patients had an acute attack of gout.

Although the serum potassium level did not change significantly in either group, the serum sodium level was significantly lower in the olmesartan plus diuretic group than in the olmesartan plus CCB group (Table 2).

Figure 3 shows the changes of estimated glomerular filtration rate throughout the study period and at the end of follow-up in the two groups. The time course of estimated glomerular filtration rate was significantly reduced in the olmesartan plus diuretic group compared with the olmesartan plus CCB group $(P<0.001)$.

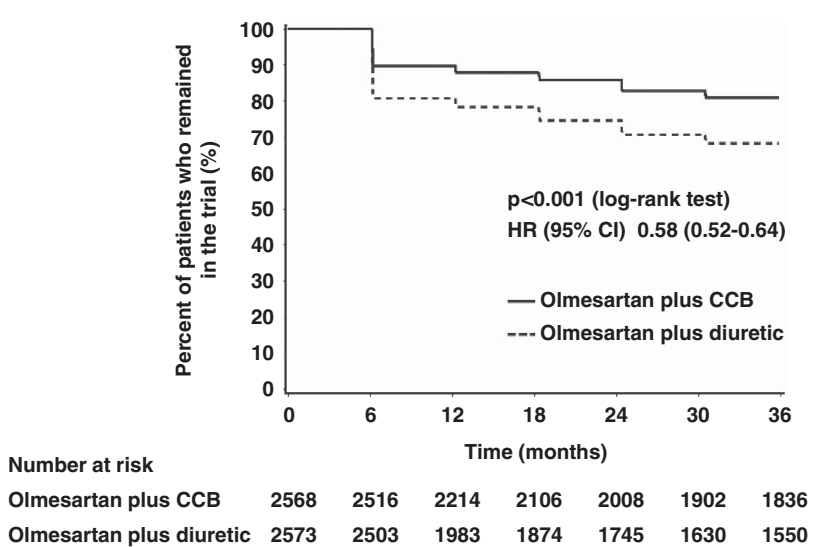

Figure 2 Treatment continuation rate during the study. 
Table 1 Serious adverse events and drug-related serious adverse events

\begin{tabular}{|c|c|c|c|c|c|c|}
\hline & \multicolumn{3}{|c|}{ Serious adverse events } & \multicolumn{3}{|c|}{ Drug-related serious adverse events } \\
\hline & $\begin{array}{l}\text { Olmesartan plus CCB } \\
\qquad(\mathrm{N}=2568)\end{array}$ & $\begin{array}{c}\text { Olmesartan plus } \\
\text { diuretic }(\mathrm{N}=2573)\end{array}$ & P-value & $\begin{array}{l}\text { Olmesartan plus } C C B \\
\qquad(\mathrm{~N}=2568)\end{array}$ & $\begin{array}{c}\text { Olmesartan plus } \\
\text { diuretic }(\mathrm{N}=2573)\end{array}$ & P-value \\
\hline Malignancy & $63(2.5)$ & $80(3.1)$ & 0.17 & & & \\
\hline Gastrointestinal disorder & $29(1.1)$ & $27(1.1)$ & 0.79 & $1(0.04)$ & $1(0.04)$ & 1.0 \\
\hline Infection & $24(0.9)$ & $22(0.9)$ & 0.76 & & & \\
\hline Fracture & $22(0.9)$ & $10(0.4)$ & 0.034 & & & \\
\hline Arrhythmia & $16(0.6)$ & $18(0.7)$ & 0.86 & $2(0.1)$ & $2(0.1)$ & 1.0 \\
\hline Death of unknown cause (except for sudden death) & $9(0.4)$ & $12(0.5)$ & 0.66 & & & \\
\hline Adverse effects on glucose metabolism & $10(0.4)$ & $10(0.4)$ & 1.0 & & & \\
\hline Bone and joint impairment & $11(0.4)$ & $8(0.3)$ & 0.50 & & & \\
\hline Syncope and dizziness & $8(0.3)$ & $11(0.4)$ & 0.64 & & & \\
\hline Renal dysfunction & $11(0.4)$ & $7(0.3)$ & 0.35 & $2(0.1)$ & $6(0.2)$ & 0.28 \\
\hline Respiratory disorder & $10(0.4)$ & $5(0.2)$ & 0.20 & & & \\
\hline Miscellaneous & $46(1.8)$ & $76(3.0)$ & 0.008 & $5(0.2)$ & $16(0.6)$ & 0.026 \\
\hline Total & $211(8.2)$ & $253(9.8)$ & 0.046 & $9(0.4)$ & $22(0.9)$ & 0.029 \\
\hline
\end{tabular}

Data are shown as number of patients (\%), several patients had two or three adverse events. CCB, calcium channel blocker.

Table 2 Biochemical variables at the baseline and at the end of study

\begin{tabular}{|c|c|c|c|c|c|c|c|}
\hline & \multicolumn{3}{|c|}{ Olmesartan plus CCB } & \multicolumn{3}{|c|}{ Olmesartan plus diuretic } & P-value \\
\hline Sodium (mEq $\mathrm{I}^{-1}$ ) & $141 \pm 2.3$ & $140 \pm 3.2$ & $-0.3 \pm 3.2$ & $141 \pm 2.6$ & $140 \pm 3.6$ & $-0.6 \pm 3.8$ & 0.038 \\
\hline Potassium (mEq $\mathrm{I}^{-1}$ ) & $4.2 \pm 0.4$ & $4.2 \pm 0.4$ & $0.02 \pm 0.47$ & $4.1 \pm 0.4$ & $4.2 \pm 0.4$ & $0.04 \pm 0.51$ & 0.31 \\
\hline Uric acid $\left(\mathrm{mg} \mathrm{dl}^{-1}\right)$ & $5.5 \pm 1.3$ & $5.6 \pm 1.4$ & $0.04 \pm 1.2$ & $5.5 \pm 1.3$ & $5.8 \pm 1.3$ & $0.2 \pm 1.3$ & $<0.001$ \\
\hline HDL cholesterol (mg dl $\left.{ }^{-1}\right)$ & $55.5 \pm 16.2$ & $56.4 \pm 15.5$ & $0.9 \pm 12.6$ & $55.6 \pm 15.9$ & $55.9 \pm 15.8$ & $0.3 \pm 13.0$ & 0.17 \\
\hline Triglyceride $\left(\mathrm{mg} \mathrm{dl}^{-1}\right)$ & $142 \pm 76.2$ & $134 \pm 71.8$ & $-8.2 \pm 74.3$ & $139 \pm 78.1$ & $134 \pm 74.0$ & $-5.1 \pm 81.2$ & 0.28 \\
\hline Creatinine $\left(\mathrm{mg} \mathrm{dl}^{-1}\right)$ & $0.79 \pm 0.24$ & $0.84 \pm 0.36$ & $0.05 \pm 0.25$ & $0.80 \pm 0.22$ & $0.89 \pm 0.42$ & $0.09 \pm 0.33$ & $<0.001$ \\
\hline
\end{tabular}

Data are mean \pm s.d., P-value for change in mean value between the two groups.

\section{【eGFR】}

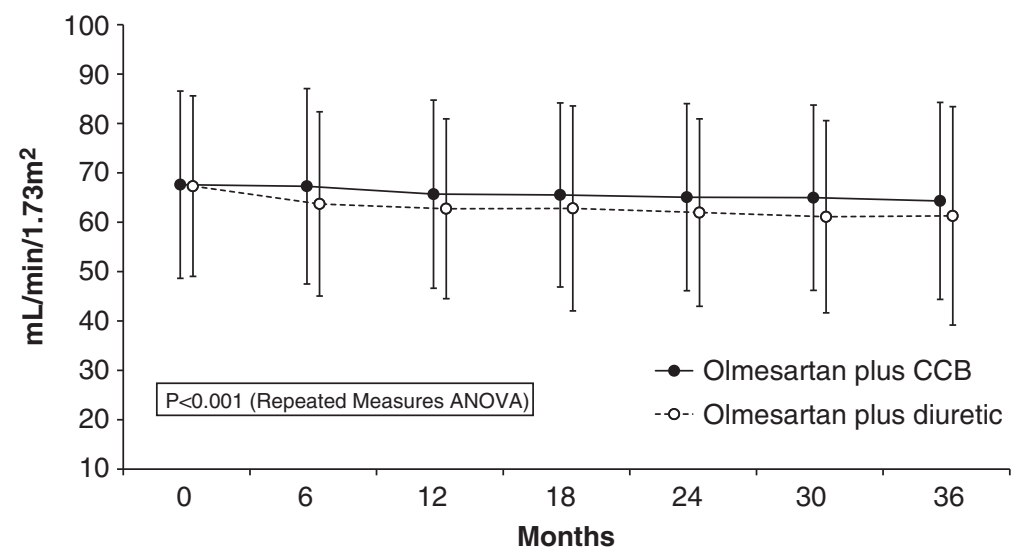

Number of subjects

$\begin{array}{lrrrrrrr}\begin{array}{l}\text { Olmesartan } \\ \text { plus CCB }\end{array} & 1852 & 1956 & 1863 & 1684 & 1609 & 1549 & 1542 \\ \begin{array}{l}\text { OImesartan } \\ \text { plus diuretic }\end{array} & 1866 & 1940 & 1839 & 1644 & 1586 & 1520 & 1520\end{array}$

Figure 3 Changes of eGFR during the study. eGFR, estimated glomerular filtration rate. 


\section{DISCUSSION}

Total discontinuation rate, incidences of AEs, SAEs, drug-related SAEs and discontinuation due to SAEs were lower in the olmesartan plus $\mathrm{CCB}$ group than in the olmesartan plus diuretic group.

In the ACCOMPLISH study, the total study drug discontinuation rate was similar for both the treatment groups, being $28.8 \%$ for the benazepril plus amlodipine group and $31.2 \%$ for the benazepril plus hydrochlorothiazide group. In contrast, the total study drug discontinuation rate was significantly lower for the olmesartan plus CCB group than the olmesartan plus diuretic group in the present study $(P<0.001)$.

In a double-blind comparison of $\mathrm{CCB}$ alone with diuretic, in Japanese elderly hypertensive patients, 6 out of 204 in patients with CCB and 9 out of 210 patients with diuretic discontinued treatment because of SAEs. ${ }^{11}$

Laboratory abnormalities, such as elevation of uric acid, elevation of creatinine and a decrease of sodium, were more common in the olmesartan plus diuretic group than in the olmesartan plus CCB group and might have contributed to the higher incidence of SAEs in the olmesartan plus diuretic group. Concerning the lower incidence of fracture in the olmesartan plus diuretic group, treatment with a thiazide diuretic may have had a role because these diuretics decrease urinary excretion of calcium and influence bone metabolism, ${ }^{12}$ although this could have been a chance finding.

Even with low-dose diuretic therapy, elevation of serum uric acid could not be avoided. In our previous study of combined treatment with hydrochlorothiazide (12.5 mg) and the ARB (losartan) for 8 weeks, the uric acid level increased significantly despite the uricosuric action of losartan. ${ }^{13-15}$ Therefore, an increase of uric acid cannot be avoided by combining a thiazide diuretic with any type of ARB. Several studies have shown that the serum uric acid level is a predictor of cardiovascular events. ${ }^{16-19}$

The significant reduction of estimated glomerular filtration rate caused by the combination of olmesartan and a diuretic during the early treatment period was probably related to the volume reduction induced by the diuretic.

Although there is a well-known relationship between the thiazide dose and changes in serum potassium, glucose and uric acid levels, ${ }^{20}$ there was no significant difference in hypokalemia between the olmesartan plus CCB group and the olmesartan plus diuretic in the present study. Therefore, it seems that the combination of olmesartan plus a low-dose thiazide diuretic may not increase the risk of new-onset of diabetes. In fact, the combination of a thiazide diuretic and an angiotensin converting enzyme (ACE) inhibitor or an ARB is widely used clinically and appears to be associated with less risk of diabetes than combined therapy with a beta-blocker ${ }^{5}$ or other antihypertensive drugs.

There were several limitations of the present study. First, this study used the PROBE method which has the potential drawback of investigator bias. Even though the endpoints, including the safety endpoints were reviewed by a blinded Endpoint committee, biased reporting of endpoints (particularly AEs) could possibly have occurred. However, BP control was similar in the two groups and it is unlikely that the PROBE design affected the main study outcomes. In addition, the sample size may not have been large enough. However, the actual incidence of primary endpoints was close to the expected rate of events, as shown in the design paper.

In conclusion, $\mathrm{ARB}$ plus $\mathrm{CCB}$ therapy was superior to the ARB plus diuretic therapy with regard to occurrence of AEs and study drug discontinuation.

\section{CONFLICT OF INTEREST}

All authors report receiving lecture fees and/or research funding from various pharmaceutical companies in Japan, including Daiichi Sankyo Co. Ltd.

\section{ACKNOWLEDGEMENTS}

We thank the collaborators and members of the COLM study group. The present study was funded by Japan Heart Foundation. This study was endorsed by the Japanese Society of Hypertension. The COLM study was funded by a grant from the Japan Heart Foundation.

1 Collins R, Peto R, MacMahon S, Hebert P, Fiebach NH, Eberlein KA, Godwin J, Qizillbash N, Taylor JO, Hennekens $\mathrm{CH}$. Blood pressure, stroke, and coronary heart disease. Part 2, Short-term reductions in blood pressure: overview of randomized drug trials in their epidemiological context. Lancet 1990; 335: 827-838.

2 ALLHAT Officers and Coordinators for the ALLHAT Collaborative Research Group. Major outcomes in high-risk hypertensive patients randomized to angiotensin-converting enzyme inhibitor or calcium channel blocker vs diuretic: The Antihypertensive and Lipid-Lowering Treatment to Prevent Heart Attack Trial (ALLHAT). JAMA 2002; 288: 2981-2997.

3 Jamerson K, Weber MA, Bakris GL, Dahlof B, Pitt B, Shi V, Hester A, Gupte J, Gatlin M, Velazquez EJ, for the ACCOMPLISH Trial Investigators. Benazepril plus amlodipine or hydrochrolothiazide for hypertension in high-risk patients. N Engl J Med 2008; 369: 2417-2428.

4 Gradman AH, Basile JN, Carter BL, Bakris GL, American Society of Hypertension Writing Group. Combination therapy in hypertension. J Am Soc Hypertens 2010; 4: 42-50.

5 Matsuzaki M, Ogihara T, Umemoto S, Rakugi H, Matsuoka H, Shimada K, Abe K, Suzuki N, Eto T, Higaki J, Ito S, Kamiya A, Kikuchi K, Suzuki H, Tei C, Ohashi Y, Saruta $\mathrm{T}$, Combination Therapy of Hypertension to Prevent Cardiovascular Events Trial Group. Prevention of cardiovascular events with calcium channel blocker-based combination therapies in patients with hypertension: a randomized controlled trial. J Hypertens 2011; 29: 1649-1659.

6 Agarwal R, Weir MR. Blood pressure response with fixed-dose combination therapy: comparing hydrochlorothiazide with amlodipine through individual-level meta-analysis. J Hypertens 2013; 31: 1692-1701.

7 Ogihara T, Matsuzaki M, Umemoto S, Rakugi H, Matsuoka H, Shimada K, Higaki J, Ito S, Kamiya A, Suzuki H, Ohashi Y, Shimamoto K, Saruta T, Combination Therapy of Hypertension to Prevent Cardiovascular Events Trial Group. Combination therapy for hypertension in the elderly: a sub-analysis of the Combination Therapy of Hypertension to Prevent Cardiovascular Events (COPE) Trial. Hypertens Res 2012; 35: 441-448.

8 Ohishi M, Kawai T, Hayashi N, Kitano S, Katsuya T, Nagano M, Hirotani A, Yamamoto K, Kamide K, Rakugi H. Effect of tablets with a combination of telmisartan and amlodipine on patients with hypertension: the Cotalo study. Hypertens Res 2013; 36: 620-626.

9 Ogihara T, Saruta T, Rakugi H, Saito I, Shimamoto K, Matsuoka H, Shimada K, Ito S, Horiuchi M, Imaizumi T, Takishita S, Higaki J, Katayama S, Kimura G, Umemura S, Ura N, Hayashi K, Odawara M, Tanahahi N, Ishimitsu T, Kashihara N, Morita S, Teramukai S. for the COLM Investigators Combinations of olmesartan and a calcium channel blocker or a diuretic in elderly hypertensive patients: a randomized, controlled trial. J Hypertens 2014; 32: 2054-2063.

10 Ogihara T, Saruta T, Rakugi H, Shimamoto K, Ito S, Matsuoka H, Horiuchi M, Imaizumi T, Takishita S, Higaki I, Katayama S, Saito I, Shimada K, on behalf of the COLM study investigators. Rationale, study design and implementation of the COLM study: the combination of olmesartan and calcium channel blocker or diuretic in highrisk elderly hypertensive patients. Hypertens Res 2009; 32: 163-167.

11 National Intervention Cooperative Study in Elderly Hypertensives Study Group. Randomized double-blind comparison of a calcium antagonist and a diuretic in elderly hypertensives. Hypertension 1999; 34: 1129-1133.

12 La Croix AZ, Ott SM, Ichikawa L, Scoles D, Barlow WE. Low-dose hydrochlorothiazide and preservation of bone mineral density in order adults. Ann Intern Med 2000; 133: 516-526.

13 Nakashima M, Uematsu T, Kosuge K, Kanamaru M. Pilot study of the uricosuric effect of DuP-753, a new angiotensin II receptor antagonist, in healthy subjects. Eur J Clin Pharmacol 1992; 42: 333-335.

14 Soffer BA, Wright JT Jr, Pratt JH, Wiens B, Goldberg AI, Sweet CS. Effects of losartan on a background of hydrochlorothiazide in patients with hypertension. Hypertension 1995; 26: 112-117.

15 Saruta T, Ogihara T, Matsuoka H, Suzuki H, Toki M, Hirayama Y, Nonaka K, Takahashi $K$. Antihypertensive efficacy and safety of fixed-dose combination therapy with losartan plus hydrochlorothiazide in Japanese patients with essential hypertension. Hypertens Res 2007; 30: 729-739.

16 Franse LV, Pahor M, Bari MD, Shorr RI, Wan JY, Somes GW, Applegate WB. Serum uric acid, diuretic treatment and risk of cardiovascular events in the Systolic Hypertension in the Elderly Program (SHEP). J Hypertens 2000; 18: 1149-1154.

17 Wang JG, Staessen JA, Fagard RH, Birkenhager WH, Gong L, Liu L, for the Systolic Hypertension in China (Syst-China) Trial Collaborative Group. Prognostic significance of serum creatinine and uric acid in older Chinese patients with isolated systolic hypertension. Hypertension 2001; 37: 1069-1074. 
18 Holeggens A, Alderman MH, Kjeldsen S, Julius S, Devereua RB, De Faire U, Fyhrquist $F$, Ibsen $H$, Kristianson $K$, Lederballe-Pedersen $O$, Lindholm $L H$, Nieminen MS, Omvik P, Oparil S, Wedel H, Chen C, Dahlof B, for the LIFE Study Group. The impact of serum uric acid on cardiovascular outcomes in the LIFE study. Kidney Int 2004; 65: 1041-1049.

19 Ito S, Naritomi H, Ogihara T, Shimada K, Shimamoto K, Tanaka H, Yoshiike N. Impact of serum uric acid on renal function and cardiovascular events in hypertensive patients treated with losartan. Hypertens Res 2012; 35: 867-873.

20 Law MR, Wald NJ, Morris JK, Jordan RE. Value of low dose combination treatment with blood pressure lowering drugs: analysis of 354 randomized trials. BMJ 2003; 326 $1427-1431$. (c) (i) $(9)$ This work is licensed under a Creative Commons Attribution-NonCommercial-NoDerivs 3.0 Unported License. The images or other third party material in this article are included in the article's Creative Commons license, unless indicated otherwise in the credit line; if the material is not included under the Creative Commons license, users will need to obtain permission from the license holder to reproduce the material. To view a copy of this license, visit http://creativecommons.org/licenses/by-nc-nd/3.0/ 